\title{
An Enhanced Underwater Linear Wireless Sensor Network Deployment Strategy for Data Collection
}

\author{
Zahoor Ahmed \& Kamalrulnizam Abu Bakar \\ PCRG Lab, Faculty of Computing \\ Universiti Teknologi Malaysia \\ 81310 UTM Johor Bahru, Johor, Malaysia \\ Email: zahoorahmad390@gmail.com, knizam@utm.my
}

Submitted: 12/01/2018. Revised edition: 11/06/2018. Accepted: 26/06/2018. Published online: 21 November 2018

\begin{abstract}
The deployment of Linear Wireless Sensor Network (LWSN) in underwater environment has attracted several research studies in the underwater data collection research domain. One of the major issues in underwater data collection is the lack of robust structure in the deployment of sensor nodes. The challenge is more obvious when considering a linear pipeline that covers hundreds of kilometers. In most of the previous works, nodes are deployed not considering heterogeneity and capacity of the various sensor nodes. This lead to the problem of inefficient data delivery by the sensor nodes on the underwater pipeline to the sink node at the water surface. Therefore, in this study, an Enhanced Underwater Linear Wireless Sensor Network Deployment (EULWSND) strategy has been proposed in order to improve the robustness in linear sensor underwater data collection. To this end, this paper presents a review of related literature in an underwater linear wireless sensor network. Further, a deployment strategy is discussed considering linearity of the underwater pipeline and heterogeneity of sensor nodes. Some research challenges and directions are identified for future research work. Furthermore, the proposed deployment strategy is implemented using AquaSim and compared with an existing data collection scheme. The results demonstrates that the proposed EULWSND outperforms the existing Dynamic Address Routing Protocol for Pipeline Monitoring (DARP-PM) in terms of overhead and packet delivery ratio metrics. The scheme performs better in terms of lower overhead with $17.4 \%$ and higher packet delivery with $20.5 \%$.
\end{abstract}

Keywords - Linear sensor network, Data collection, Underwater, Pipeline, Wireless sensors, Deployment

\section{INTRODUCTION}

The surveillance of underwater pipeline has become a crucial issue because of importance of the crude oil transported via the pipeline. Because, high natural resources of a nation such as crude oil is transported using the pipe, which can pass through large sea or Ocean [1]. Therefore, it is paramount to monitor the condition of the pipeline in the case of oil leakage due to burst of pipeline or even due to external attack. This has led to the employment of wireless sensor network for pipeline condition's data collection for pipeline monitoring. Several studies have been suggested in solving communication issues among underwater sensing devices and development of underwater communication devices [2]. The key purpose of Underwater Wireless Sensor Network (UWSN) is to offer a low cost and an unmanned system for the underwater applications such as surveillance, oil and gas pipeline monitoring, pollution monitoring, offshore exploration, survey and underwater reservoir haunting [3]. The underwater surroundings is harsh in nature therefore, the underwater communication is much difficult and expensive using sound waves as a means of communication. It is obvious that the sound waves transmission is being used naturally to pass information among underwater living creatures [4]. UWSN transmission is also based on acoustic channels, which often need to look at the main issues of acoustic signals comprising (a) Regular movement of the water that affects acoustic signal and increase bit error rates. (b) Propagation delay, which is five times, more than the radio frequency, (c) Reduction of signal strength is observed more in underwater communication. These key issues, severely affects the efficiency of acoustic transmission. Although, UWSN has some research challenges and has a subarea known as the Underwater Linear Wireless 
Sensor Network (ULWSN), which involves monitoring of linear structures such as the underwater pipeline network. In the underwater surroundings, maintaining pipeline's condition is an important task, which requires active and consistent monitoring process [5]. However, this kind of monitoring procedure is very capital intensive because of the high cost of tools, technicians, or robot systems, especially in a remote and difficult underwater environment. Fig. 1 depict the illustration of the underwater pipeline with sensors.

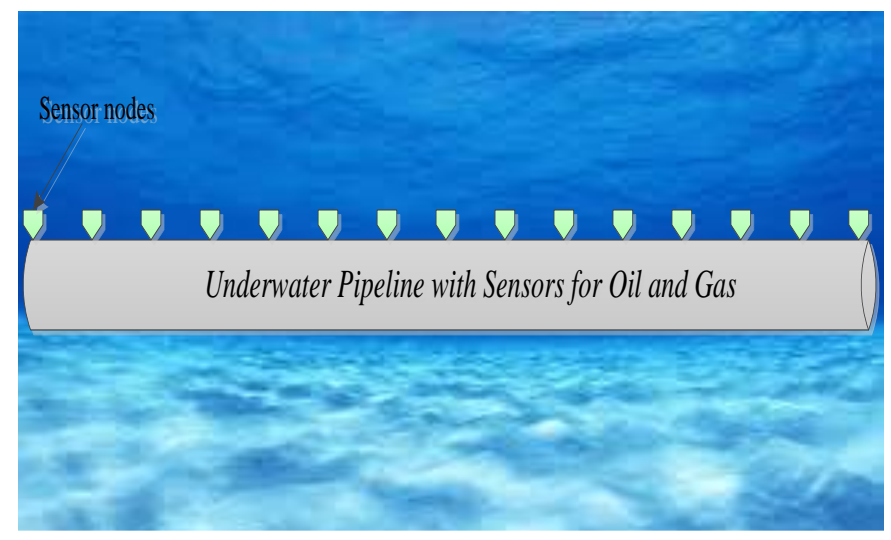

Fig. 1. Underwater Pipeline with Sensors

Additionally, the network structure and topology for data collection has a major impact on the performance of the network. In fact, the way and manner of the design of any network have a major effect on the network output [6]. There are two different deployment approaches in underwater sensor network namely, dense sensors network and sparse sensors network deployment. The dense sensors deployments are mainly employed when there is a large number of sensors to be considered for small coverage area while the sparse sensors deployment approach employs high capability sensors placed at a longer distance to cover a large area.

Normally, Sensor Nodes (SNs) are mounted in a pipeline network field to monitor different parameters regarding the pipeline and its surroundings, also, it is assumed that sensors are installed in a very remote area, in such a harsh condition and left unattended [7]. The connection between the SNs and Autonomous Unmanned Vehicles (AUVs) as well as the connection between AUVs and the sink is often an acoustic communication. The connection between the sink and Network Control Centre (NCC) can use any type of communication technologies. The installation of a fixed communication structure in a remote field to gather data from the sensors is prohibitive and impractical because of random structure of the installation environment. Therefore, in such situations, ad-hoc sensor network in combination with AUV layered approach can be beneficial in collecting data from underwater surroundings. Consequently, the linear sensor deployment strategy needs to be further explored.

In this paper, a critical review of different underwater linear sensors networks scheme and deployment has been conducted. An enhanced node deployment structure based on heterogeneity of sensors has been put forth. Further, some research issues and research directions have been highlighted. Finally, implementation of the strategy is carried out and benchmarked.

The remaining path of the paper is structured as follows. Section II entails the review of related studies in an underwater linear wireless sensor network. Section III involves the discussion of the deployment strategy for linear wireless sensor networks. Section IV presents the results and its analysis. Section V highlights some research issues and future research directions. Finally, Section VI concludes the research study.

\section{RElAted Literature IN UNDERWATER PIPELINE DATA COLLECTION}

In this section, the detailed review of related literature on the underwater linear sensor network data collection are discussed. The related literature has been categorized into three (3) including chain based data collection, distributed topologybased data collection and Autonomous Unmanned Vehicle (AUV)-based data collection.

\section{A. Chain-Based Data Collection Approach}

In this subsection, underwater data collection, which is based on chain linear arrangement, is explained. In chain-based nodes deployment schemes, all the nodes must be connected in a linear direction like chain rings as depicted in Fig. 2, which demonstrates the chain based deployment model. There is a high level of reliability risk if these nodes are connected through a wire such as if any of the nodes stops working, all the connected nodes will be out of service. It is also possible to decrease the risk by making the floatable-mobile chain like [8].

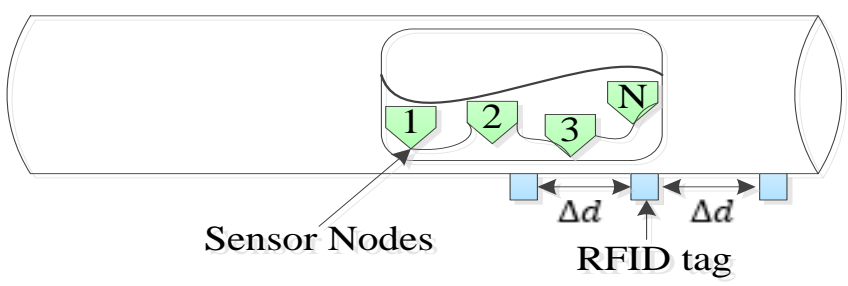

Fig. 2. Chain Based Deployment Model

\section{B. Distributed Topology-Based Data Collection Approach}

In this subsection, the non-centralized data collection approaches are explained. Linear Sensor Network consists mainly of the thin, thick and very thick types of deployment schemes, depending on how the sensor nodes are deployed, either in one line or in multiple lines. In the distributed topology, data collection scheme in Jawhar et al., [9] for LSNs creates an ordered list of the nodes in the network according to their approximate position. This list will maintain the linear topology of the network and assist in the coordination of linearly deployed nodes. Routing tables can be constructed on each node by using the details in this list. The efficiency, 
reliability, and robustness of the routing process can also be improved. In addition, failure in the nodes can be detected and energy consumption can be minimized.

Distributed Underwater Clustering Scheme (DUCS) in Domingo and Prior, [10] is a dynamic scheme that can calculate and divide the entire network into clusters and the distributed algorithm used to manage these clusters. Each local cluster will need to select cluster head prior to starting a communication with other nodes. The data are collected hierarchal from non-cluster head nodes towards their respective cluster heads. This transmission is directly between the ordinary sensors and cluster heads in a single hop. Thereafter, the cluster heads will obtain the aggregate of the received data and forward to the sink by using multi-hop routing. The cluster heads play an intermediary role and transparently controls the intra-cluster and inter-cluster communication. In addition, cluster heads can be changed and rotated in the cluster according to their remaining energy. DUCS works in two steps. The first step is called the set-up phase, where the entire network is divided into clusters, and in the second phase, the data communication will be handled.

\section{Autonomous Unmanned Vehicle-Based Data Collection Approach}

In this subsection, the autonomous unmanned vehicle-based data collection approaches have been discussed.

Autonomous systems are unique in their functionalities and they need a path for the movement of autonomous devices/robots. Kim et al., [11] mentioned SPAMMS as a system, which has a robot agent-based sensing technology for the monitoring of linear structures like pipelines. SPAMMS use RFID technique for the deployment of mobile sensors and autonomous robots. The mobile sensors are deployed in very important locations of the pipelines and the inspection can be scheduled and performed based on demand. When a mobile sensor is deployed in a pipeline and starts to function, it can move with the fluid flowing inside the pipeline. The fixed sensors convey event position details to mobile sensors for tracking of the damaged location in the pipeline. After the analysis of events reports, a robot agent travel inside the pipelines to perform detailed examination and maintenance of the reported incidents such as damage, leakage, or corrosion of the pipeline. In addition, in Jawhar et al., [12] a framework for employing unmanned aerial vehicle for data gathering in linear wireless sensor network has been suggested.

In the proposed work of Heredia et al., [13], a multi UAVs framework was presented. This method increased the dependability of Unmanned Aerial Vehicle (UAV) sensor Fault Detection and Identification (FDI). In addition, the Differential Global Positioning System (DGPS) and inertial sensors are used for sensor FDI in each UAV. Furthermore, an extraordinary position estimation algorithm, which boosts individual UAV FDI system used. Additional estimations are obtained using images received from two different UAVs while in an active state. However, the accuracy and noise level of the estimation depends on factors such as dynamic re- planning of the paths for different UAVs that obtain a better estimation in a situation where the faults caused by the errors in absolute position estimation cannot be detected using local FDI in the UAVs. In addition, the two main UAVs are discussed, and different data collection scenarios are highlighted. The Fig. 3 represents AUV based data collection illustration in underwater.

The effect of an undetected oil spillage can be disastrous. Therefore, it is important to effectively monitor the pipeline systems. For this reason, many researchers have utilized the Unmanned Aerial Vehicles (UAV) and this has resolved the problem of optimal pipeline monitoring using multiple mobile nodes. Ondráček et al., [14] have provided a mathematical model, which captures the properties of the problem, including the environmental sensitivity and the path planning constraints of UAVs. In addition, two algorithms are designed to enhance the inherent scalability limits of this problem. The researchers were able to find an optimal solution for a large-scale realworld monitoring problem and provide a promising solution to UAVs path planning in such scenarios.

The Linear Wireless Sensor Network (LWSN) technology has evolved rapidly in recent years. In this connection, sensor nodes are constantly used in sensing, processing, storage, and communication processes. For LWSNs that are used in environmental, commercial and military applications, the sensors are lined up in single or multiple lines because of the maintenance of the linear structure or area that is being monitored making a special class of these LSNs. The study in Jawhar et al., [12] has highlighted the classification of various types of LSNs, they include, the multi-hop approach to collect the data all through the linear networks for linear structures such as pipeline monitoring, border monitoring, roads monitoring.

Usually, these structures are extended for hundreds or even thousands of kilometers and increase the cost in the form of energy consumption. To reduce energy utilization in data collection in order to increase the network lifetime, this research presents a framework for monitoring linear infrastructures using LSNs where data collection and transmission is done using Unmanned Aerial Vehicles (UAVs).

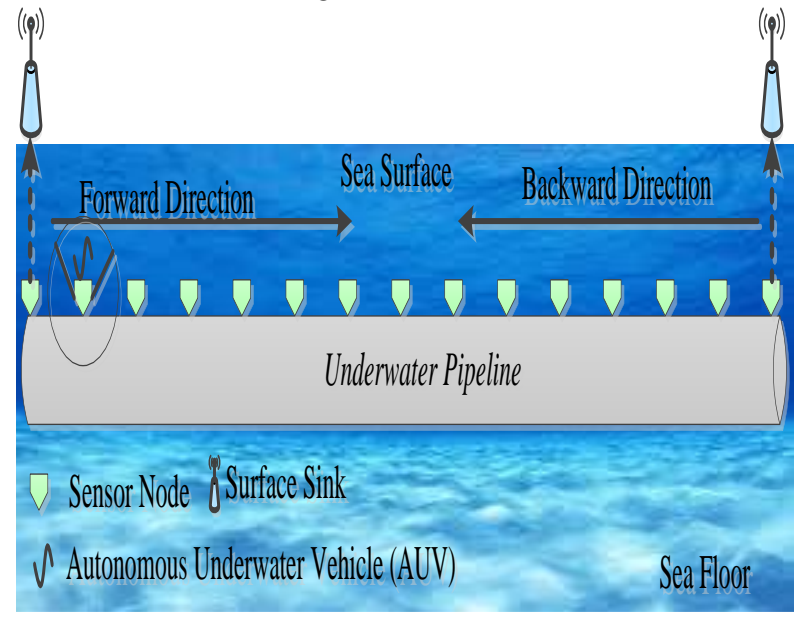

Fig. 3. AUV Based Data Collection 
The system consists of four types of nodes, which includes sensor nodes (SNs), relay nodes (RNs), UAVs, and sinks. A UAV moves back and forth along the linear network and transports the data that is collected by the RNs to the sinks located at both ends of the LSN. This network architecture is known as a UAV based LSNs (ULSNs). Using this approach, the node energy consumption can be reduced because of the significant reduction in the transmission ranges of the $\mathrm{SN}$ and $\mathrm{RN}$ nodes and the use of a one-hop transmission to communicate the data from the RNs to the UAV. In addition, the three types of UAV movement approaches are discussed, simulated, and analyzed to measure system performance under various network conditions.

The multi-hop routing approach is expensive to be used for the long-range LSN because it may utilize more energy and decrease the lifetime of the entire network. An effective solution for the long-range LSN deployment is to use the AUV approach in Jawhar et al., [15], which can minimize the transmission range and energy consumption of the basic sensors and increase the lifetime of the network. Using this approach, the AUV periodically hover around the sensing nodes and collects data from the BSN directly. Further, the AUV can move in a different direction to improve monitoring coverage. Apart from the AUV, the mobile sensor nodes are also commonly used for the monitoring of linear structures such as the long-range water pipelines, this system is designed by deploying mobile wireless sensor nodes inside the pipeline and these nodes will coordinate with each other in accordance with the application requirement. The nodes moves in groups and only one node actived at a time. The wakeup mechanism assists in changing the state of the nodes and conserve energy. This work can be evaluated by estimating the energy consumption and memory utilization. A related example of this type of work is in [16]. With all the related studies, it can be concluded that there is a need for an improvement in the deployment of the linear wireless sensor network.

\section{ENHANCED UNDERWATER LINEAR WIRELESS SENSOR NETWORK DEPLOYMENT STRATEGY}

In this section, the improved concept of Enhanced Underwater Linear Wireless Sensor Network Deployment (EULWSND) has been presented and discussed.

In our proposed strategy, static sensor nodes are deployed on the underwater pipeline network and AUVs are used to collect data from these sensors. Sensor Nodes (NS) are deployed to monitor different parameters of the pipeline. These sensor nodes do not know their position, the position of AUV and the route of AUV. The uniqueness of this work is that heterogeneity and capability with their coverage area are considered in these proposed studies. In order to efficiently deploy the sensor nodes, the following operations are carried out. Fig. 4 depicts the illustration for the linear sensor node deployment. The thin layer topology distribution and five levels data communication have been considered in the pipeline node deployment as seen in the figure below.

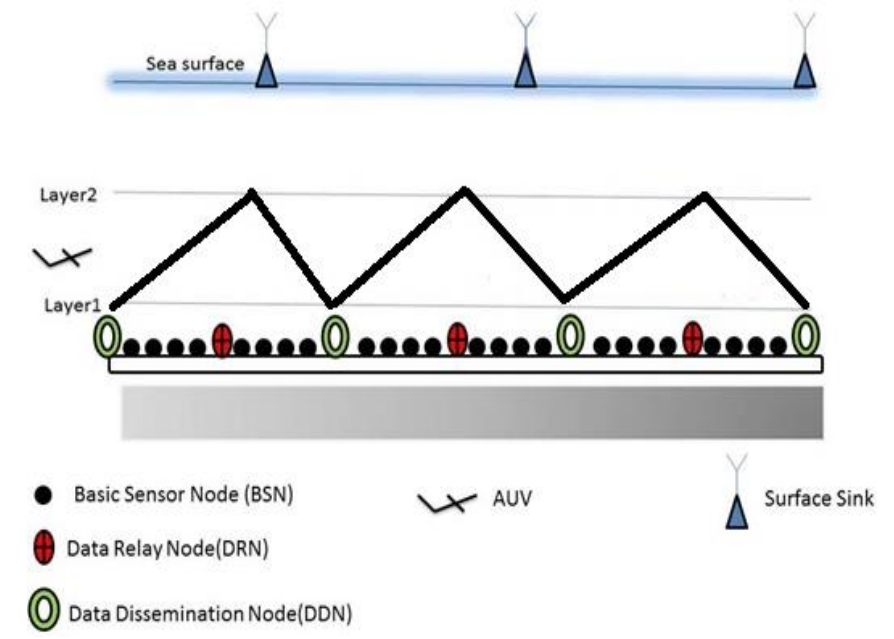

Fig. 4. Illustration of the Linear Sensor Network Structure

A. Steps in Node Deployment

- Basic sensor are hybrid in nature

- Calculate the distance and location position of each type of sensor node to be installed on the linear pipeline.

- Position the nodes named as Basic Sensor Nodes (BSN) based on estimated distance interval. The BSN nodes only forward data to the DRN.

- Place the Data Relay Node (DRN) in between the BSN based on approximated distance. The DRN forwards data collected to the DDN for aggregation and subsequent forwarding.

- Then situate the Data Dissemination Node (DDN) in between a longer range of BSN nodes also based on calculated distance. The DDN only communicate with AUV, which hovers across the pipeline for data collection purpose and is sent to the sink the NOC (Network Operation Center).

- Design and develop an AUV path planning as part of the deployment strategy.

- The AUV path planning hovered across the pipeline in a sinusoidal form, collecting data only from the DDNs. This data are transmitted to the sink nodes at sea surface

- The AUV is calculated using the following formula in Eq. (1)

$$
y_{p}=R_{D}-T_{0}+d_{p}\left[\sin ^{2}\left(\frac{\pi x}{R_{S}}\right)\right]
$$

The $R_{D}$ is the signal coverage of a range of DDN. Where $d_{p}$ is the distance between two DDN. The $T_{0}$ is the small amplitude projection of the AUV across both the DDN and sinks' signal coverage area. The path planning for the AUV is represented as $y_{p}$. Coverage signal range for sink node is $R_{s}$.

Considering all these steps involved in the deployment process an efficient and minimum energy consumption will be 
achieved in the underwater linear wireless sensor networks Fig. 5 depicts the hierarchy deployment of the linear sensor node.

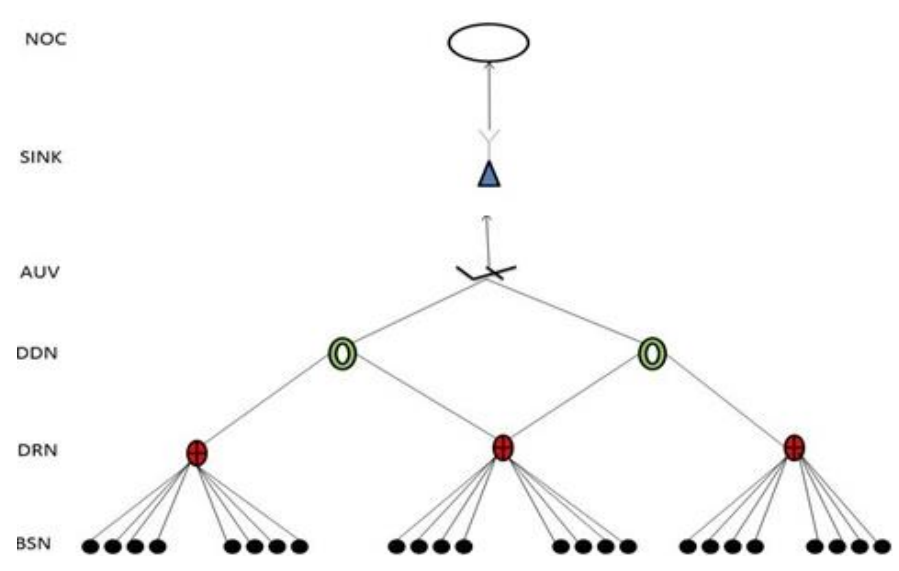

Fig. 5. Proposed Hierarchical Network Structure

B. Algorithm for the deployment strategy

Begin

Initialize $B S N, D R N, D D N$ and $A U V$ path

Set a counter for the different nodes

For every $100 \mathrm{~m}$ deploy BSN

If the distance is certain

Else for $500 \mathrm{~m}$ deploy $D R N$

Else for $2500 \mathrm{~m}$ deploy $D D N$

\section{Endif}

Set AUV and estimate it path

Calculae AUV Postion Path $=R_{D}-T_{0}+d_{p}\left[\sin ^{2}\left(\frac{\pi x}{R_{g}}\right)\right]$

\section{Stop deployment process}

\section{End}

The pipelines sensors nodes including BSN, DRN, and DDN with AUVs and sinks collects data hierarchically according to Fig. 5 hierarchical model. The BSNs performs the basic sensing function while, DRN nodes collect data from BSNs and afterward, forwards this data to DDNs which in turn, forwards the data to AUVs and finally, data is received at the sink.

The Algorithm shows the basic steps in the deployment of the linear sensor network networks in the pipeline application. The next section presents the implementation and result of the proposed strategy.

\section{EXPERIMENTATION AND RESULT DISCUSSION}

In this section, the simulation experiments and result discussion are presented. The proposed Enhanced Underwater Linear Wireless Sensor Network Deployment (EULWSND) strategy is simulated using AQUASIM NS-2. The result of the implementation is compared against the DARP-PM protocol. The evaluation is based on communication overhead and packet delivery ratio. The Table I represents the simulation parameters considered.

TABLE I. SIMULATION PARAMETERS

\begin{tabular}{|l|l|}
\hline \multicolumn{1}{|c|}{ Parameters } & \multicolumn{1}{c|}{ Values } \\
\hline MAC & Underwater MAC \\
\hline Transport layer protocol & UDP \\
\hline Antenna & Directional \\
\hline Propagation Model & $\begin{array}{l}\text { Underwater } \\
\text { Propagation }\end{array}$ \\
\hline AUV Speed & $0.1-0.3$ \\
\hline Channel Bandwidth & $25 \mathrm{Khz}$ \\
\hline Number of Sinks & 5 \\
\hline Number of nodes & $20-200$ \\
\hline Network dimension & $12600 \mathrm{~m} \times 500 \mathrm{~m}$ \\
\hline Primary sinks location & $\begin{array}{l}\text { At both end of the } \\
\text { pipeline }\end{array}$ \\
\hline Types of nodes & BSN, DRN, DDN \\
\hline Ranges of nodes & $100,250,400,500 \mathrm{~m}$ \\
\hline $\begin{array}{l}\text { Maximum Pipeline } \\
\text { length }\end{array}$ & $90000 \mathrm{~m}$ \\
\hline Hello packet size & 12 byte \\
\hline Simulation time & $1000 \mathrm{sec}$ \\
\hline
\end{tabular}

The table depicts all parameters considered and their corresponding values. We now discussed the results obtained based on the metrics considered.

\section{A. Communication Overhead}

The average communication overhead of the packet during data collection is evaluated. The proposed EULWSND outperforms the existing baseline studies that is, DARP-PM as shown in Fig. 6. The proposed enhanced linear sensor deployment performs better because of their less communication between the nodes. In our deployment strategy, the nodes are placed by estimating different transmission capabilities of the heterogeneous sensors. This strategy adopted enables fewer control packets communication. Thus, there is lower communication overhead compared with the existing protocol.

\section{B. Packet Delivery Ratio}

In this subsection, the Packet Delivery Ratio (PDR) is evaluated during the data collection process. In addition, the proposed deployment strategy provides better performance result when compared to the same DARP-PM protocol. Higher packet delivery is achieved because this strategy does 
not overwork the nodes and hence there is a higher delivery of packet since more powerful heterogeneous sensor nodes are integrated into the deployment design and development. The packet delivery is improved due to the efficient coverage of the under pipeline area because higher capacity sensors are utilized. The result is displayed in Fig. 7. For a clear understanding of the performance gain of the two compared protocols.

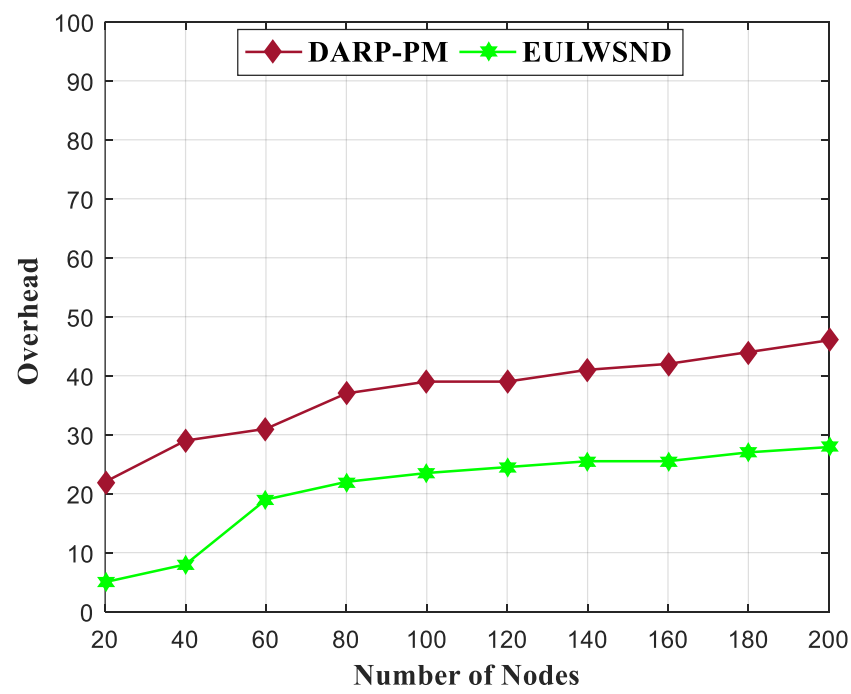

Fig. 6. Communication Overhead Result

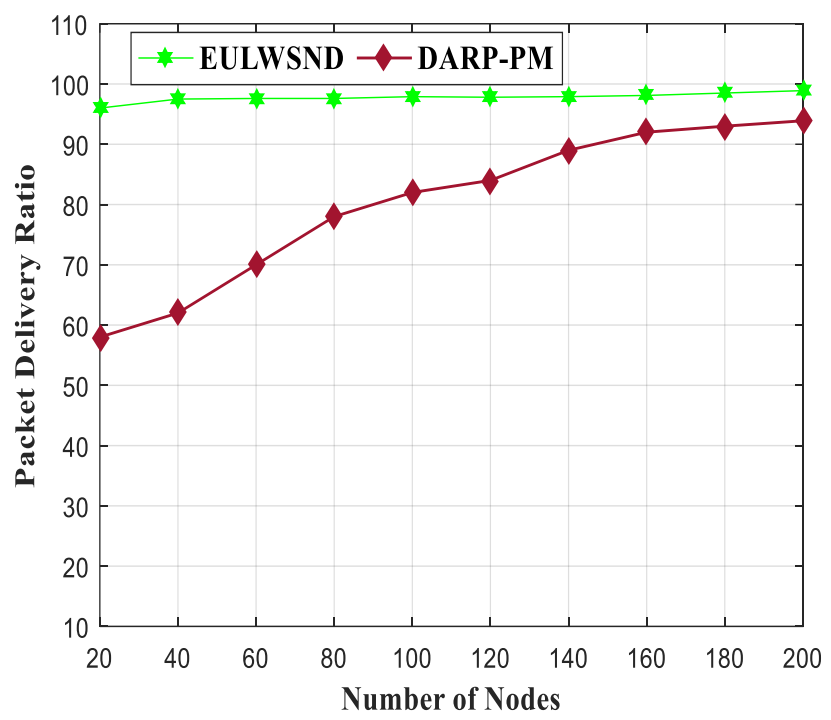

Fig. 7. Packet Delivery Ratio Result

\section{OPEN RESEARCH ISSUES AND CHALLENGES}

In this section, the issues related to linear sensor network for underwater pipeline monitoring are highlighted and discussed as follows.

- Heterogeneous sensor nodes have been considered for efficient data collection. In future research, there is need to consider the cost-effectiveness of the sensor nodes and the AUVs in the linear network.

- The optimization technique might have a direct impact on the overall performance of the data collection scheme. In the future research work, the presented algorithm in APPER can be extended by employing optimization technique such as Non-dominant Sorting Genetic Algorithm II (NSGA-II), Particle Swamp Optimization (PSO) and distributed learning automation.

- Delay sensitivity method for the linear sensor network data collection is very important to achieve a more efficient delivery of data from the base of the pipeline to the sink at the surface of the water. Thus, it would be necessary to take benefit of the delay sensitive scheme in order to improve the data collection process.

The mentioned issues needs to be addressed in the future research work.

\section{CONCLUSION}

The proposed Enhanced Underwater Linear Wireless Sensor Network Deployment (EULWSND) strategy provides a robust deployment of sensor nodes in linear pipeline monitoring. This study presented a node deployment strategy for efficient data collection. The proposed sensor node deployment strategy has been compared with an existing baseline research work, namely DARP-PM. The proposed EULWSND strategy outperforms the baseline work (DARPPM). The EULWSND obtain lower communication overhead with approximately $17.4 \%$ decrease. In addition, the EULWSND gain $20.5 \%$ increase in terms of packet delivery ratio better than the DARP-PM protocol. Considering the results mentioned earlier, the proposed strategy has demonstrated its strength and efficiency for data collection in an underwater linear sensor network.

\section{REFERENCES}

[1] Pipeline Guerrillas. (2007). Offshore Technology. Available online.

[2] Ayaz, M., Baig, I., Abdullah, A., \& Faye, I. (2011). A Survey on Routing Techniques in Underwater Wireless Sensor Networks. Journal of Network and Computer Applications, 34(6), 1908-1927.

[3] Jawhar, I., Mohamed, N., \& Agrawal, D. P. (2011). Linear Wireless Sensor Networks: Classification and Applications. Journal of Network and Computer Applications, 34(5), 16711682.

[4] Forrest, T. G. (1994). From Sender to Receiver: Propagation and Environmental Effects on Acoustic Signals. American Zoologist, 34(6), 644-654.

[5] BenSaleh, M. S., Qasim, S. M., Obeid, A. M. and Garcia-Ortiz, A. (2013). A Review on Wireless Sensor Network for Water Pipeline Monitoring Applications. Collaboration Technologies and Systems (CTS), 2013 International Conference on (128-131) IEEE. 
[6] Smith, V. A., Jarvis, E. D. and Hartemink, A. J. (2002) Influence of Network Topology and Data Collection on Network Inference. Biocomputing 2003, 164-175.

[7] Ali, S., Qaisar, S. B., Saeed, H., Khan, M. F., Naeem, M. and Anpalagan. (2015). A Network Challenges for Cyber Physical Systems with Tiny Wireless Devices: A Case Study on Reliable Pipeline Condition Monitoring. Sensors, 15(4), 7172-7205.

[8] Kim, J., Lim, J. S., Friedman, J., Lee, U., Vieira, L., Rosso, D., et al. (2009). SewerSnort: A Drifting Sensor for In-Situ Sewer Gas Monitoring. Paper presented at the Sensor, Mesh and Ad Hoc Communications and Networks, 2009. SECON'09. 6th Annual IEEE Communications Society Conference on.

[9] Jawhar, I., Mohamed, N., \& Zhang, L. (2012). A Distributed Topology Discovery Algorithm for Linear Sensor Networks. Paper presented at the Communications in China (ICCC), 2012 1 st IEEE International Conference on.

[10] Domingo, M. C., \& Prior, R. (2007). A Distributed Clustering Scheme for Underwater Wireless Sensor Networks. Paper presented at the Personal, Indoor and Mobile Radio Communications, 2007. PIMRC 2007. IEEE 18th International Symposium on.

[11] Kim, J.-H., Sharma, G., Boudriga, N., \& Iyengar, S. S. (2010). SPAMMS: A Sensor-based Pipeline Autonomous Monitoring and Maintenance System. Paper presented at the Communication Systems and Networks (COMSNETS), 2010 Second International Conference on
[12] Jawhar, I., Mohamed, N., Al-Jaroodi, J., \& Zhang, S. A. (2014). Framework for Using Unmanned Aerial Vehicles for Data Collection in Linear Wireless Sensor Networks. Journal of Intelligent \& Robotic Systems, 74(1-2) 437.

[13] Heredia, G., Caballero, F., Maza, I., Merino, L., Viguria, A., \& Ollero, A. (2009). Multi-unmanned Aerial Vehicle (UAV) Cooperative Fault Detection Employing Differential Global Positioning (DGPS), Inertial and Vision Sensors. Sensors, 9(9): 7566-7579.

[14] Ondráček, J., Vaněk, O., \& Pěchouček, M. (2014, June). Monitoring Oil Pipeline Infrastructures with Multiple Unmanned Aerial Vehicles. International Conference on Practical Applications of Agents and Multi-Agent Systems. Springer International Publishing, 219-230.

[15] Jawhar, I., Mohamed, N., Al-Jaroodi, J., \& Zhang, S. (2013). An Efficient Framework for Autonomous Underwater Vehicle Extended Sensor Networks for Pipeline Monitoring. Paper presented at the Robotic and Sensors Environments (ROSE), IEEE International Symposium on.

[16] Almazyad, A. S., Seddiq, Y. M., Alotaibi, A. M., Al-Nasheri, A. Y., BenSaleh, M. S., Obeid, A. M., et al. (2014). A Proposed Scalable Design and Simulation of Wireless Sensor NetworkBased Long-Distance Water Pipeline Leakage Monitoring System. Sensors, 14(2), 3557-3577. 